\title{
Palatal Volume Changes in Unilateral Cleft Lip and Palate Paediatric Patients
}

\author{
Valentina PUCCIARELLI ${ }^{1}$, Luca PISONI ${ }^{1}$, Marcio DE MENEZES ${ }^{2}$, Ana Maria CERON-ZAPATA ${ }^{3}$, \\ Ana Maria LOPEZ-PALACIO ${ }^{3}$, Marina CODARII ${ }^{1}$, Chiarella SFORZA ${ }^{1 *}$ \\ ${ }^{1}$ Dipartimento di Scienze Biomediche per la Salute, Università degli Studi di Milano, Milano, Italy; \\ ${ }^{2}$ School of Health Science, State University of Amazonas, Brazil; ${ }^{3}$ University CES. Medellin, Colombia
}

DOI: $10.15221 / 15.139 \quad$ http://dx.doi.org/10.15221/15.139

\begin{abstract}
Cleft lip and/or palate (CL/P) are the most common craniofacial abnormalities. CL/P therapy involves also orthopedic and surgical treatments. In particular, the orthopedic treatment can help to align the cleft segments and facilitate surgery. Traditionally, facial and palatal structures have been analyzed by 2D methods, omitting information of volume bone defects. A pre-surgical volume estimation can be useful to determine the anatomical boundaries of the alveolar bone defect; subsequently, volume assessments can appreciate the outcome of secondary alveolar bone grafting. In the present study, we developed a 3D stereophotogrammetric technique for volume estimation of the dental arches of children with $\mathrm{CL} / \mathrm{P}$. The method was employed to assess the 3D changes occurring in the maxillary arch of unilateral CLP (UCLP) patients with the use of plates before the first year of life. We collected 96 palatal casts of 32 neonatal patients with UCLP. Palatal casts were obtained before orthopedic treatment, before cheiloplasty, and after cheiloplasty. Half patients were treated with an active plate, half with a passive one. Casts were marked with a set of landmarks, digitized using a stereophotogrammetric system, and then analyzed. Volumes of the greater and the minor alveolar segments were separately assessed, and compared with a 3-w repeated measures ANOVA. Method accuracy was assessed using objects of known size, while repeatability was evaluated using Student's $t$ test and technical error of measurements. Volume estimates were accurate, without systematic errors; random errors were lower than $5 \%$ of the total variance. Significant effects of alveolar segment and time were observed $(p<0.0001)$. Instead, no differences were found for the kind of plate. In conclusion, stereophotogrammetric systems can be a valid instrument to estimate palatal volumes of patients with CL/P during treatment, that can be followed up in a safe, rapid and non-invasive way.
\end{abstract}

Keywords: Unilateral cleft lip and palate, stereophotogrammetry, palatal casts

\section{Introduction}

Cleft lip and cleft palate are among the most common craniofacial congenital deformities. The malformation occurs between the $1^{\text {st }}$ and the $3^{\text {rd }}$ months of intrauterine life, and involves still unknown mechanisms of cell migration in the maxillary, fronto-nasal and palatal processes of the developing embryo and fetus. It can be an isolated deformity (non-syndromic clefts, $60-70 \%$ of cases), or within a combined set of alterations (syndromic clefts). The non-syndromic cleft lip and/ or palate (CL/P) affects 1 in each 700 livebirths, with different percentages in the various ethnic groups [1].

The patients may present with a variety of clefts, ranging from cleft lip, cleft lip and palate, or isolated cleft palate; the alteration can be unilateral or bilateral. Depending on the complexity of the pathology, $\mathrm{CL} / \mathrm{P}$ patients require a multidisciplinary approach in order to ameliorate functional, social and psychological aspects of their life. Generally, patients are longitudinally submitted to several types of treatments, comprising orthopedic and surgical procedures [2,3]. In particular, the orthopedic treatment, performed with active and passive acrylic plates, can help to align the cleft segments and facilitate surgery [4-6].

Patient care begins at birth, and the suitable treatments are determined by diagnosis, monitoring growth and defining the effect of rehabilitation. Therefore, analyzing palatal morphology before, during, and at the end of the treatment becomes essential for a good clinical prognosis.

Traditionally, palatal skeletal structures have been analyzed by two-dimensional (2D) methods, such as periapical, occlusal, and panoramic radiographs [7-9], omitting information about the volume of the alveolar bone defects. Indeed, a pre-surgical volume estimation can be useful to determine the anatomical boundaries of the alveolar bone defect; subsequently, volume assessments can appreciate the outcome of secondary alveolar bone grafting $[7,10,11]$.

\footnotetext{
* chiarella.sforza@unimi.it; +39 02503 15387; www.scibis.unimi.it
} 
Some investigations proposed the use of computed tomography to estimate the alveolar bone defects in CL/P patients [7,10], but the practical, clinical application of the method is limited by the amount of ionizing radiations given to the subjects, especially when longitudinal examinations in growing patients are needed. More recently, palatal volume estimations of these patients have been performed by using cone beam computed tomography [11], with a reduced radiation exposure. Unfortunately, the method is still invasive, and it cannot be applied to all children that should be followed up.

In contrast, different methods of three-dimensional (3D) imaging are being used for the assessment of facial soft tissues and dental arches in CL/P individuals, which represents the first stage in diagnosis. Apart from with volumetric methods, like computed tomography and magnetic resonance imaging, optical surface methods, like digital dental casts, stereophotogrammetry, and laser scanning, are the most frequently used 3D techniques in CL/P patients to evaluate treatment effects and changes over time $[5,9,12-15]$. Optical methods can also be employed to estimate the volume of structures: in particular, nasal volumes had been recently obtained in CL/P patients [16].

Among the various methods, stereophotogrammetry has been described as accurate, repeatable and non-invasive, well suitable for the analysis of facial soft tissues $[16,17]$. Recently, it has also been successfully used for assessing the stone casts of newborn patients with CL/P $[18,19]$.

Indeed, dental casts can be digitized, and their virtual copies be used to measure several palatal characteristics in 3D $[5,9,14,18,20,21]$. The method offers a noteworthy change in stone cast data collection and analysis: alongside with accurate measurements, the technology provides easy storage and time saving, permitting a virtual planning and assessment of the medical procedures and treatments that can be performed at distance reducing costs and increasing efficacy even in those countries with a lower technological development [15].

In particular, data from stone casts of CL/P patients could show the correlation of the cleft defect to the alveolar bone and palatal size disparities. The method developed in our laboratory allowed the quantitative assessment of longitudinal modifications in palatal dimensions and surface area, permitting to follow-up the modifications in the absolute and relative dimensions of the alveolar segments during treatment $[18,19]$.

In the present study, we developed a 3D stereophotogrammetric technique for volume estimation of the dental arches of children with CL/P. Accuracy and repeatability of the method were tested. Additionally, the method was used to assess the 3D changes occurring in the maxillary arch of unilateral cleft lip and palate (UCLP) patients submitted to orthopedic treatment with the use of nasoalveolar molding (NAM) and Hotz's plate [6] prior to rhinocheiloplasty and before the first year of life.

\section{Method}

\subsection{Experimental Design}

The present study comprised two separate phases: at first, we devised a 3D stereophotogrammetric technique for volume estimation of the dental arches of newborn children with CL/P. Accuracy (also called "trueness"), that is closeness of agreement between the actual test results and a true or golden standard reference value, and repeatability (or "precision"), that is closeness of agreement between repeated test results of the method, were tested [19].

The second phase is part of a longitudinal clinical study performed to evaluate the 3D morphological effects of various treatments on the growing segments of dental arches of patients with UCLP younger than one year of age not subjected to early surgical procedures or prior orthopedic treatment [6].

\subsubsection{Patients}

Thirty-two neonatal patients (15 girls and 17 boys) with UCLP attending the Fundacion Clınica Noel de Medellın (Colombia) were examined. The ethics committee of Universidad CES (Medellin, Colombia) evaluated and approved the study (File 22, September 2008). All procedures, possible discomforts and risks, together with possible benefits, were fully explained to the parents of children, who signed an informed consent form. They were free to exit from the study at any time.

Impressions of the dental arches were taken at three different time points during follow up: A) prior to presurgical orthopedic treatment (mean age 10.5 days, SD 4.8), B) before cheiloplasty (mean age 83.3 days, SD 6.6) and C) after cheiloplasty (mean age 317.1 days, SD 44.2). Impressions were cast in dental stone; a total of 96 palatal cast models was available for the study [18]. 
Presurgical orthopedic treatment was performed with either passive or active plates [6]. For passive plate treatment, Hotz's plate was used (10 boys, 6 girls). For active plate treatment, Presurgical Naso Alveolar Molding (PNAM) was employed ( 7 boys, 9 girls). The casts were numbered, and coded A, B, $\mathrm{C}$ according to the relevant time point. All subsequent measurements were made blindly to the kind of used plate, while the information about the time point was available to the investigators.

\subsubsection{Dental cast digitization}

The method for dental cast digitization has already been described in detail $[18,19]$. In brief, a commercial 3D stereophotogrammetry system (VECTRA-3D, Canfield Scientific, Inc., Fairfield, NJ, USA) was used, and digital reproductions of the casts were obtained. The files were analyzed using the stereophotogrammetric software (Mirror imaging software, Canfield Scientific, Inc., Fairfield, NJ, USA).

Figure 1 shows the three palatal casts of one patient with right side UCLP, one for each time point. The greater (right side in this case) and minor (left side) cleft segments can be appreciated.

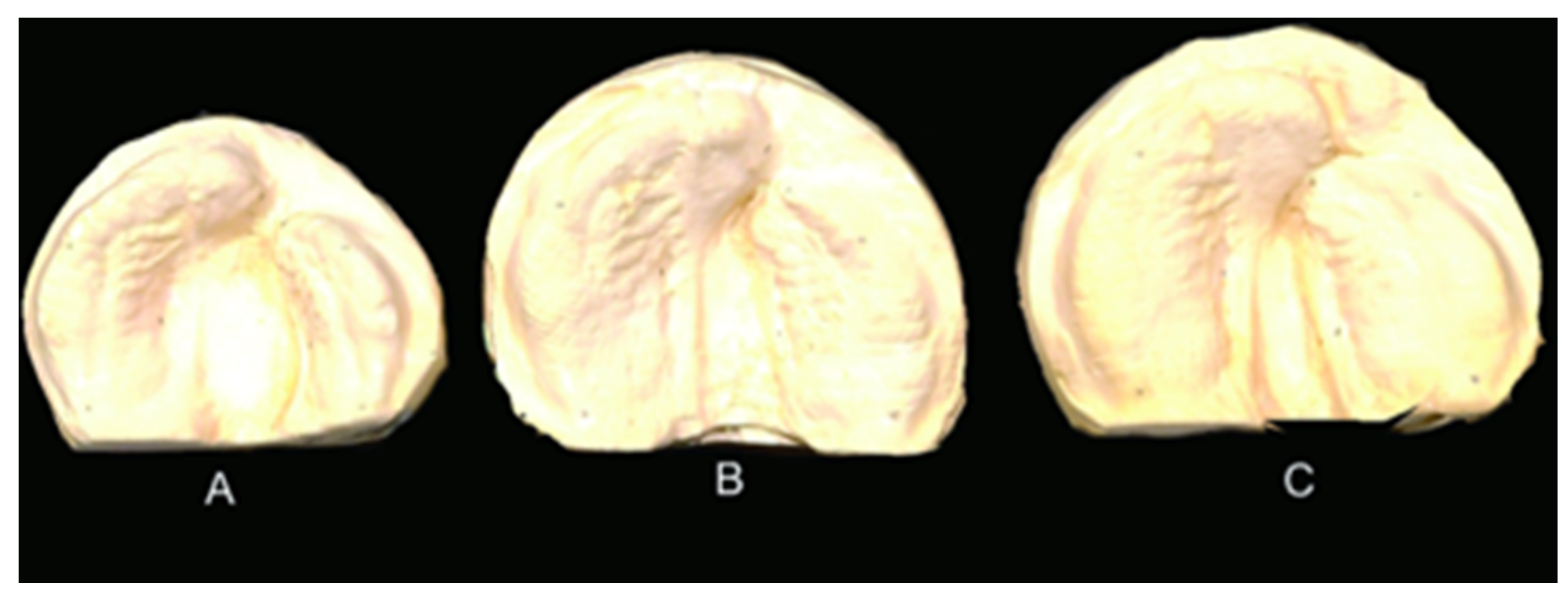

Fig. 1. Palatal casts digitized with stereophotogrammetry at time points $A, B$, and $C$.

\subsection{Measurements}

\subsubsection{System accuracy and repeatability (volume)}

Previous investigations performed in our laboratory had already found that the stereophotogrammetric system can assess the coordinates of soft tissue facial landmarks with good precision and reproducibility, without systematic errors among operators, calibration steps, and repeated acquisitions [17]. Additionally, we have already found that it can measure linear distances, angles and areas of objects of known dimension and simple geometry (cubes and cylinders of different sizes) with an accuracy error lower than $0.9 \%$. No systematic errors between operators' measurements were found [18].

Accuracy of area measurements was also tested directly on the palatal segments of UCLP dental models using small strips of millimeter paper glued across the two cleft segments. For both cleft segments, no significant differences were found between the actual and the measured values; mean absolute differences were lower than $1 \mathrm{~mm}^{2}$, and errors lower than $2 \%$ [19]. A method for the assessment of the actual area of the greater and minor segments was devised, and found to the repeatable, with mean absolute differences of $0.1 \mathrm{~mm}^{2}$, random errors lower than $3.2 \mathrm{~mm}^{2}$, and no intra- and inter-operator differences [19].

In the current study, we tested accuracy and repeatability of volume assessments. To assess method accuracy, a set of objects of known size and simple geometry (pyramids of different dimensions) was digitized using the stereophotogrammetric instrument, data were saved and analyzed using the processing software of the instrument. The actual (true) volume of the objects was obtained from their linear dimensions. Accuracy errors (AE, unit: percentage) were used to compare the measurements with the reference values.

$$
\mathrm{AE}=\frac{\text { true value }- \text { measured value }}{\text { true value }} \times 100
$$


To evaluate repeatability, measurements were repeated by both the same and different operators in two separate occasions. Paired Student's $t$ tests and technical errors of measurement (TEM, or Dahlberg's error) were used to assess systematic and random errors respectively. The percentage of variance explained by the method error was computed as the ratio between squared TEM and squared standard deviation multiplied by 100 .

$$
\mathbf{T E M}=\sqrt{\sum \frac{(\text { value } \mathbf{1}-\text { value } \mathbf{2})^{2}}{2 n}}
$$

\subsubsection{Palatal volumes}

To estimate the volumes of the greater and minor cleft segments, at first the method devised by De Menezes et al. [19] and Pisoni [22] for area measurements was used. In brief, using the software tools of the stereophotogrammetric instrument, the base of each segment was outlined with a set of 20-30 points. The points were positioned at the boundary between the alveolar process and the maxillary bone; their actual number depended from the segment dimensions (Fig. 2). The total area of each alveolar segment was then obtained (Fig. 3).

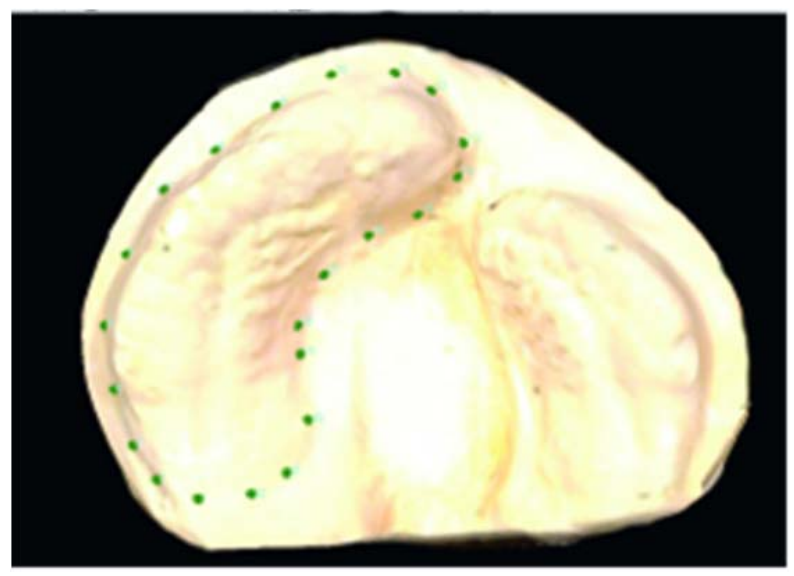

Fig. 2. The base of the greater cleft segment is outlined by a set of 22 points.

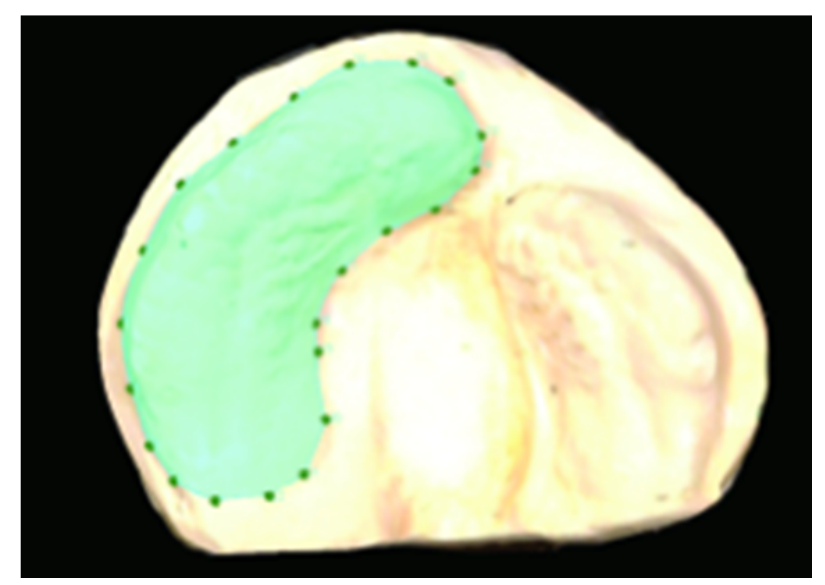

Fig. 3. The segment area is coloured in light blue.

A virtual plane was created and imported inside the dental cast file. For each alveolar segment, the points were then projected on this plane and both objects were associated using the registration tool of the stereophotogrammetric software (Mirror imaging software, Canfield Scientific, Inc., Fairfield, NJ, USA), as illustrated in Figure 4. Registration of the two objects (dental cast and virtual plane) was made using the points with the same number. In this way, the virtual plane "cuts" the cast at the base of the alveolar process, and the volume between the two objects can be calculated using the appropriate tool of the software [22]. The procedure was made separately for both alveolar segments (Fig. 5). 


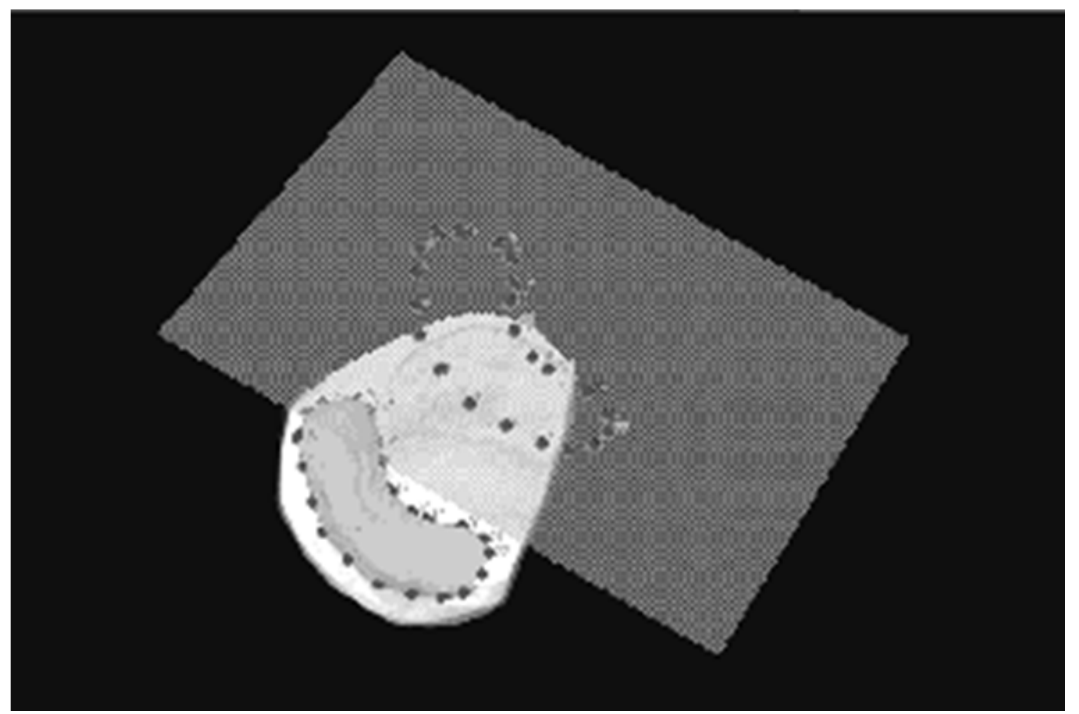

Fig. 4. Projection of the points outlining the border of the area on the virtual plane.

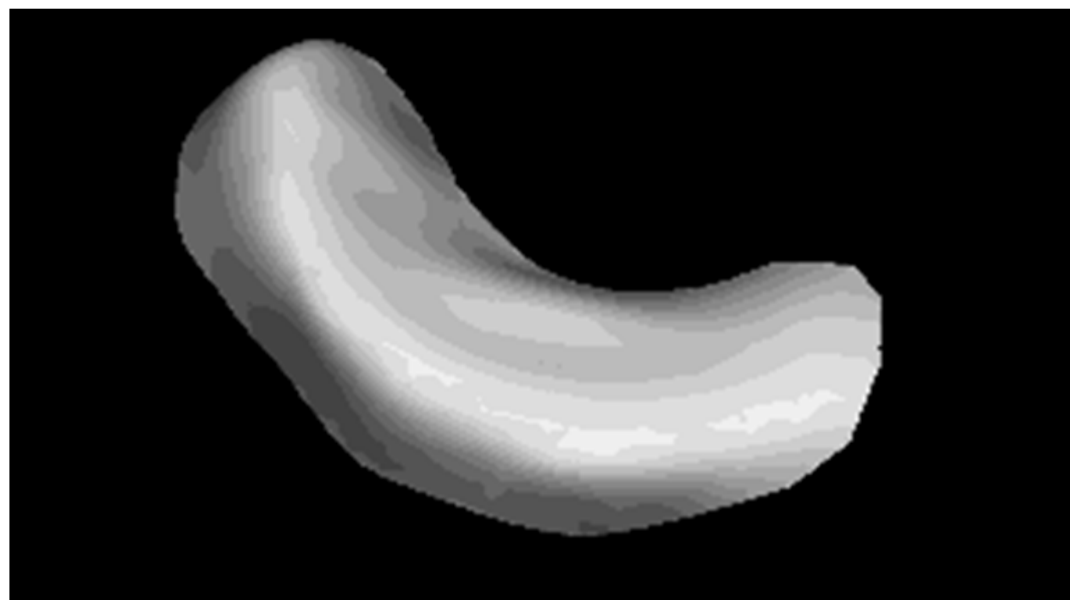

Fig. 5. Final step of the procedure: the alveolar process is isolated from the cast, and its volume is calculated.

\subsection{Statistical analysis}

The repeatability of volume measurements of the palatal segments was made on a subset of 20 casts, randomly selected from the complete set of 96 casts. Different operators and the same operator over time calculated the volume of the segments. Data were analyzed using paired Student's tests, and TEMs were computed. In addition, the mean absolute difference (MAD, average of absolute differences between the values of two sets of measurements) was calculated.

Descriptive statistics were obtained for each segment (greater and minor), time point (prior to presurgical orthopedic treatment; before cheiloplasty; after cheiloplasty), and kind of plate (passive, Hotz's plate; active, PNAM plate).

To compare the volume measurements between the two types of plates, and to compare each type of plate at the three different time points, a three-factor repeated measures analysis of variance (ANOVA) was used to determine differences between plate (between subjects factor), alveolar segment (within subject factor), and time (within subject factor). The interactions between factors were calculated. For each comparison, the statistical power was also computed. Post-hoc tests were made where appropriate. Box's Test of Equality of Covariance Matrices and Levene's Test of Equality of Error Variances found that the homoscedasticy hypothesis was verified in all occasions.

Significance level was set at $5 \%$ for all analyses. 


\section{Results}

\subsection{System accuracy and repeatability}

Table 1 reports the volume measurements obtained on the objects of known size and simple geometry. The differences between measurements obtained on the geometric objects were quite low, with $A E$ lower than $0.5 \%$. No significant differences were found between the true and the measured volumes ( $p$ $>0.05$ ), and the method error explained less than $3 \%$ of the total variance.

Table 1. Accuracy of the proposed method for volume estimation.

\begin{tabular}{|l|l|l|}
\hline & Object 1 & Object 2 \\
\hline True value $\left(\mathrm{cm}^{3}\right)$ & 145.6 & 269.8 \\
\hline Measured value $\left(\mathrm{cm}^{3}\right)$ & 146.3 & 269.1 \\
\hline Accuracy error $(\%)$ & 0.47 & 0.27 \\
\hline
\end{tabular}

Cleft segment volume measurements obtained in two different occasions by the same operator and between different operators had no systematic errors $(p>0.05)$. Low TEM values were found for both intra-operator (TEM $=0.02 \mathrm{~cm}^{3}$, percentage of explained variance $2 \%$ of the total variance) and inter-operator (TEM $=0.075 \mathrm{~cm}^{3}$, percentage of explained variance $3.75 \%$ ) repeated measurements. The mean absolute difference for inter-operator was lower than $0.1 \mathrm{~cm}^{3}$.

\subsection{Effect of time and treatment on palatal segments}

Descriptive statistics for palatal segment volumes in the three analyzed time points and for the two kinds of plates are listed in Table 2; the relevant statistical comparisons are reported in Table 3. No significant differences were found for the kind of plate (three-factor repeated measures ANOVA, between-subjects factor, $p>0.05$ ). The observed statistical power was reduced, showing that a notable type II error was present.

Instead, significant effects of alveolar segment and time were observed $(p<0.0001)$, with a very high statistical power. Post hoc tests found significant differences at all time points (pairwise comparisons: A vs $B, p=0.001$; $A$ vs $C, p<0.001$; $B$ vs $C, p=0.003$ ).

There was also a significant interaction between time and plate: with PNAM plate most of the time-related variations in both alveolar segments volume were between time points B (before cheiloplasty) and C (after cheiloplasty), while with Hotz's plate they were more homogenously distributed.

Table 2. Descriptive statistics of volume measurements during the follow-up. All values are $\mathrm{cm}^{3}$.

\begin{tabular}{|l|l|l|l|l|l|l|l|}
\hline Plate & & \multicolumn{2}{|c|}{ Time Point A } & \multicolumn{2}{c|}{ Time Point B } & \multicolumn{2}{c|}{ Time Point C } \\
\hline & Segment & Minor & Greater & Minor & Greater & Minor & Greater \\
\hline \multirow{2}{*}{ PNAM } & Mean & 0.53 & 1.08 & 0.48 & 1.09 & 0.65 & 1.22 \\
\cline { 2 - 8 } & SD & 0.25 & 0.47 & 0.26 & 0.49 & 0.29 & 0.56 \\
\hline \multirow{2}{*}{ Hotz's } & Mean & 0.52 & 0.91 & 0.68 & 1.09 & 0.81 & 1.38 \\
\cline { 2 - 8 } & SD & 0.21 & 0.29 & 0.23 & 0.37 & 0.25 & 0.51 \\
\hline
\end{tabular}

Table 3. Three-factor repeated measures analysis of variance.

\begin{tabular}{|l|l|l|l|}
\hline & $F$ & P value & Power \\
\hline Between subjects & & & \\
\hline Plate & 0.390 & 0.537 & 0.093 \\
\hline Within subjects & & & \\
\hline Time & 24.397 & $<0.0001$ & 0.998 \\
\hline Segment & 103.65 & $<0.0001$ & 1.000 \\
\hline Interactions & & & \\
\hline Time X Plate & 5.737 & 0.023 & 0.640 \\
\hline Segment X Plate & 1.507 & 0.229 & 0.221 \\
\hline Time X Segment & 2.090 & 0.159 & 0.288 \\
\hline Time X Segment X Plate & 1.147 & 0.293 & 0.179 \\
\hline
\end{tabular}




\section{Conclusions}

In the current investigation, we devised a method for the estimation of the volume of palatal segments from stone casts of children with UCLP using a commercial stereophotogrammetric system. The study completes our previous experiments, where the same instrument had been successfully used to assess linear distances and surface areas on the same palatal casts [18,19].

The devised technique started from the previously defined surface areas, and combined them with a virtual plane that was used to "cut" the alveolar segments. A similar procedure was devised by van Loon et al. [16] for the longitudinal analysis of nasal dimensions, but no comparisons with gold standards (or actual, true values) were reported. In our laboratory, the protocol was tested for accuracy and repeatability using objects of simple geometry, and found to be reliable. Stereophotogrammetry can therefore be used for the digitization of neonatal palates, where a reduced depth and the edentulous alveolar process combine in a favorable geometry [18,19]; in contrast, dedicated laser scans must be employed for child and adult dental arches [9].

Obviously, the current method can be applied only for structures directly visible form the external body surface, and cannot be employed to estimate the alveolar bone defects in CL/P patients, where volumetric scanners should be used $[7,10,11]$.

Subsequently, the method was used within a longitudinal clinical study which is evaluating the modifications in palatal dimensions during orthopedic treatments in patients with UCLP younger than one year of age [6]. For this second task, we found a highly significant effect of time: the longitudinal study started when the children were in their first two weeks of life, and ended at a mean age of 10 months and half. Both the physiological growth of the children, and the treatments received during the follow-up can easily explain the effect. Indeed, it has been reported that bone growth in CLP patients can be stimulated in the first year of life only [23]. The same time-related effect was found for segment area $[19,22]$.

At the same time, for both area (previous study) and volume (current study), a significant difference between the greater and the minor segments was found, as expected from the clinical characteristics of UCLP patients [1]. Both measurements yielded no significant effect of the kind of plate used by the children; more investigation is needed on this topic, considering that literature reports no definitive results about the various effects of different orthopedic treatments [6,24].

In contrast, area measurements had a marginally significant time $X$ segment interaction $[19,22]$, while volume measurements showed a significant time $X$ plate interaction. Apparently, the modifications in cleft segments reflect a complex three-dimensional re-organization of palatal structures, where modifications in shape and curvature should also be analyzed with suitable methods [25].

In conclusion, stereophotogrammetric systems can be a valid instruments to estimate palatal volumes of patients with CL/P during their first year of treatment. The children can be followed up in a safe, rapid and non-invasive way.

\section{References}

[1] JA. Freitas et al, "Rehabilitative treatment of cleft lip and palate: experience of the Hospital for Rehabilitation of Craniofacial Anomalies/USP (HRAC/USP)--Part 1: overall aspects", in Journal of Applied Oral Sciences, Vol. 20, No. 1, 2012, pp. 9-15.

[2] DG. Mosmuller et al, "Scoring systems of cleft-related facial deformities: a review of literature", in Cleft Palate Craniofacial Journal, Vol. 50, No. 3, 2013, pp. 286-296, http://dx.doi.org/10.1597/11-207.

[3] DA. Papamanou et al, "Appreciation of cleft lip and palate treatment outcome by professionals and laypeople", in European Journal of Orthodontics, Vol. 34, No. 5, 2012, pp. 553-560, http://dx.doi.org/10.1093/ejo/cir073.

[4] T. Yamada et al, "Nasolabial and alveolar morphology following presurgical orthopaedic treatment in complete unilateral clefts of lip, alveolus and palate", in Journal of Cranio-maxillofacial Surgery, Vol. 31, 2003, pp. 343-347, http://dx.doi.org/10.1016/j.jcms.2003.07.004,

[5] Q. Yu et al, "CAD presurgical nasoalveolar molding effects on the maxillary morphology in infants with UCLP", in Oral Surgery Oral Medicine Oral Pathology Oral Radiology, Vol. 116, No. 4, 2013, pp. 418-426, http://dx.doi.org/10.1016/j.0000.2013.06.032.

[6] AM. López-Palacio et al, "Nasal changes with nasoalveolar molding in Colombian patients with unilateral cleft lip and palate", in Pediatric Dentistry, Vol. 34, No. 3, 2012, pp. 239-244.

[7] M. Feichtinger et al, "Volumetric assessment of secondary alveolar bone grafting using cone beam computed tomography", in Cleft Palate Craniofacial Journal, Vol. 46, No. 5, 2009, pp. 503-511. 
[8] P. Sutthiprapaporn et al, "Evaluation of alveolar bone grafting in unilateral cleft lip and palate patients using a computer-aided diagnosis system", in Imaging Science in Dentistry, Vol. 42, No. 4, 2012, pp. 225-259, http://dx.doi.org/10.5624/isd.2012.42.4.225.

[9] MA. Kuijpers et al, "Three-dimensional imaging methods for quantitative analysis of facial soft tissues and skeletal morphology in patients with orofacial clefts: a systematic review", in PLoS One, Vol. 7, No. 4, 2014, p. e93442. http://dx.doi.org/10.1371/journal.pone.0093442.

[10]P. Picolli et al, "Assessment of alveolar defect volume in unilateral cleft lip and palate patients using a free software program", in Open Journal of Stomatology, Vol. 3, 2013, pp. 31-36, http://dx.doi.org/10.4236/ojst.2013.39A005.

[11] BW. Lynderup et al, "A novel semiautomatic technique for volumetric assessment of the alveolar bone defect using cone beam computed tomography", in Cleft Palate Craniofacial Journal, Vol. 52, No. 3, 2015, pp. 47-55, http://dx.doi.org/10.1597/13-287.

[12]VF. Ferrario et al, "Three-dimensional lip morphometry in adults operated on for cleft lip and palate", in Plastic and Reconstructive Surgery, Vol. 111, No. 7, 2003, pp. 2149-2156.

[13]K. Schwenzer-Zimmerer et al, "Systematic contact-free 3D topometry of the soft tissue profile in cleft lips", in Cleft Palate Craniofacial Journal, Vol. 45, No. 6, 2008, pp. 607-613. http://dx.doi.org/10.1597/07-116.1.

[14] BZ. Mello et al, "Evaluation of the intercanine distance in newborns with cleft lip and palate using 3D digital casts", in Journal of Applied Oral Sciences, Vol. 21, No. 5, 2013, pp. 437-442. http://dx.doi.org/10.1590/1679-775720130091.

[15] C. Sforza et al, "Soft- and hard-tissue facial anthropometry in three dimensions: what's new", in Journal of Anthropological Sciences, Vol. 91, 2013, pp. 159-184, http://dx.doi.org/10.4436/jass.91007.

[16] B. van Loon et al, "3D Stereophotogrammetric assessment of pre- and postoperative volumetric changes in the cleft lip and palate nose", in International Journal of Oral and Maxillofacial Surgery, Vol. 39, No. 6, 2010, pp. 534-540. http://dx.doi.org/10.1016/j.ijom.2010.03.022.

[17]M. de Menezes et al, "Accuracy and reproducibility of a 3-dimensional stereophotogrammetric imaging system", in Journal of Oral and Maxillofacial Surgery, Vol. 68, No. 9, 2010, pp. 2129-2135, http://dx.doi.org/10.1016/j.joms.2009.09.036.

[18] C. Sforza et al, "Evaluation of a 3D stereophotogrammetric technique to measure the stone casts of patients with unilateral cleft lip and palate", in Cleft Palate Craniofacial Journal, Vol. 49, No. 4, 2012, pp. 477-483, http://dx.doi.org/10.1597/10-207.

[19] M. De Menezes et al, "Evaluation of a 3D stereophotogrammetric method to identify and measure the palatal surface area in children with unilateral cleft lip and palate", in Cleft Palate Craniofacial Journal, 2015 Mar 20 [Epub ahead of print].

[20] LT. Huanca Ghislanzoni et al, "Evaluation of tip and torque on virtual study models: a validation study", in Progress in Orthodontics, Vol. 14, 2013, pp. 19, http://dx.doi.org/10.1186/2196-1042-14-19.

[21]A. Ugolini et al, "Dental arch response to Haas-type rapid maxillary expansion anchored to deciduous vs permanent molars: A multicentric randomized controlled trial", in Angle Orthodontist, Vol. 85, No. 4, 2015, pp. 570-576, http://dx.doi.org/10.2319/041114-269.1.

[22] L. Pisoni, Three-dimensional analysis of palate morphology in unilateral cleft lip and palate children, PhD Thesis, Università degli Studi di Milano, Italy, 2015.

[23]R. Grabowski et al, "Presurgical orthopaedic treatment of newborns with clefts--functional treatment with long-term effects", in Journal of Craniomaxillofacial Surgery, Vol. 34, Suppl. 2, 2006, pp. 34-44.

[24]MA. Papadopoulos et al, "Effectiveness of pre-surgical infant orthopedic treatment for cleft lip and palate patients: a systematic review and meta-analysis", in Orthodontics and Craniofacial Research, Vol. 15, No. 4, 2012, pp. 207-236, http://dx.doi.org/10.1111/j.1601-6343.2012.01552.x.

[25]R. Rosati et al, "The labial aging process: a surface analysis-based three-dimensional evaluation", in Aesthetic Plastic Surgery, Vol. 38, No. 1, 2014, pp. 236-341, http://dx.doi.org/10.1007/s00266-013-0227-9. 